MATHEMATICS OF COMPUTATION

Volume 76, Number 260, October 2007, Pages 1771-1786

S 0025-5718(07)01952-7

Article electronically published on May 24, 2007

\title{
TWO LOWER ORDER NONCONFORMING RECTANGULAR ELEMENTS FOR THE REISSNER-MINDLIN PLATE
}

\author{
JUN HU AND ZHONG-CI SHI
}

\begin{abstract}
In this paper, we propose two lower order nonconforming rectangular elements for the Reissner-Mindlin plate. The first one uses the conforming bilinear element to approximate both components of the rotation, and the modified nonconforming rotated $Q_{1}$ element to approximate the displacement, whereas the second one uses the modified nonconforming rotated $Q_{1}$ element to approximate both the rotation and the displacement. Both elements employ a projection operator to overcome the shear force locking. We prove that both methods converge at optimal rates uniformly in the plate thickness $t$ in both the $H^{1}$ - and $L^{2}$-norms, and consequently they are locking free.
\end{abstract}

\section{INTRODUCTION}

One benchmark problem in computational science is the Reissner-Mindlin plate (R-M hereinafter) problem. For this problem, the straight-forward approach using lower order conforming finite elements in the primal formulation faces with the locking phenomenon. This occurs when the thickness $t$ of the plate tends to zero and the problem enforces a constraint (namely, the Kirchhoff constraint). For the discrete problem, this constraint, especially for lower order elements, cannot be fully satisfied. Various methods have been proposed to weaken or overcome the locking effect since the nineties of the last century, and most of them can be regarded as reduced integration methods. Recently, the discontinuous Galerkin method [1] has also been used to design finite element methods for the R-M plate problem (see, for instance, [2, 11, 16]). One common favorable feature of these discontinuous Galerkin R-M plate elements is that all the variables share the same nodes and consequently can also be extended to shell problems.

In this paper, we propose and analyze two lower order nonconforming rectangular elements for the R-M plate model. In the first element, the usual conforming bilinear element space is chosen as the rotation space and the modified nonconforming rotated element $Q_{1}$ space ( $\mathrm{NRQ}_{1}$ element hereinafter) [13, 22, 15] as the displacement space, and the reduced integration method is used to overcome the shear force locking. The second element differs from the first one only in the approximation of the rotation; which employs the modified $\mathrm{NRQ}_{1}$ element for both components of the rotation, consequently all the variables in this method share the same nodes. Furthermore, the second finite element method enjoys the same

Received by the editor July 5, 2005 and, in revised form, May 18, 2006.

2000 Mathematics Subject Classification. Primary 65N30.

Key words and phrases. Reissner-Mindlin plate, bilinear element, rotated $Q_{1}$ element, bubble function, locking-free.

This research was supported by the Special Funds for Major State Basic Research Project.

(C)2007 American Mathematical Society 1771

Reverts to public domain 28 years from publication 
promising features as the lower order triangular elements proposed in 2, 11, 16. and therefore is possible to be generalized to the shell problems.

We conclude this introduction with a list of some basic notations used in the sequel. In Section 2, we recall the Reissner-Mindlin plate model and its mixed formulation by Brezzi and Fortin [4, and Section 3 presents our elements for the R-M plate. The equivalent formulation of the discrete problem will be given and proven in Section [4. In Section [5, we show the well-posedness of the discrete problems. This paper ends with Section 6, which is devoted to error analysis.

In the sequel, $D(\Omega)$ is the linear space of an infinitely differentiable function with compact support on $\Omega$. We use the standard notation and definition for the Sobolev spaces $\left(H^{s}(\Omega)\right)^{2}$ and $\left(H^{s}(\partial \Omega)\right)^{2}$ for $s \geq 0$; the standard associated inner products are denoted by $(\cdot, \cdot)_{s}$ and $(\cdot, \cdot)_{s, \partial \Omega}$, and their respective norms by $\|\cdot\|_{s}$ and $\|\cdot\|_{s, \partial \Omega}$. For $s=0,\left(H^{s}(\Omega)\right)^{2}$ coincides with $\left(L^{2}(\Omega)\right)^{2}$. In this case, the inner product is denoted by $(\cdot, \cdot)$. As usual, $H_{0}^{s}(\Omega)$ is a closure of $D(\Omega)$ with respect to the norm $\|\cdot\|_{s}$.

Define

$$
\hat{H}^{1}(\Omega)=\left\{v \in H^{1}(\Omega): \int_{\Omega} v d x d y=0\right\} .
$$

Finally, we use the standard differential operators:

$$
\begin{aligned}
& \nabla r=\left(\begin{array}{c}
\partial r / \partial x \\
\partial r / \partial y
\end{array}\right), \quad \operatorname{curl} p=\left(\begin{array}{c}
-\partial p / \partial y \\
\partial p / \partial x
\end{array}\right), \\
& \operatorname{div} \boldsymbol{\psi}=\frac{\partial \psi_{1}}{\partial x}+\frac{\partial \psi_{2}}{\partial y}, \quad \operatorname{rot} \boldsymbol{\psi}=\frac{\partial \psi_{2}}{\partial x}-\frac{\partial \psi_{1}}{\partial y}
\end{aligned}
$$

Throughout this paper, the generic constant $C$ is assumed to be independent of the plate thickness $t$ and the mesh size $h$.

\section{Reissner-Mindlin Plate MOdel}

In this section, we recall the widely used Reissner-Mindlin plate equations. Let $\Omega$ be the region occupied by the plate, and $\omega$ and $\phi=\left(\phi_{1}, \phi_{2}\right)$ denote the transverse dispacenent of mid-section and the rotation of the fibers normal to mid-section, repectively. The Reissner-Mindlin plate model determines $\omega$ and $\phi$ as the solution to the following variational problem,

Problem 2.1. Find $(\omega, \phi) \in H_{0}^{1}(\Omega) \times\left(H_{0}^{1}(\Omega)\right)^{2}$ such that

$$
a(\boldsymbol{\phi}, \boldsymbol{\psi})+\lambda t^{-2}(\nabla \omega-\boldsymbol{\phi}, \nabla v-\boldsymbol{\psi})=(g, v), \quad \forall(v, \boldsymbol{\psi}) \in H_{0}^{1}(\Omega) \times\left(H_{0}^{1}(\Omega)\right)^{2},
$$

where $g$ is the scaled transverse loading function, $t$ the plate thickness,

$$
\lambda=E k / 2(1+\nu)
$$

the shear modulus with E Young's modulus, $\nu$ the Poisson ratio, and $\kappa$ the shear correction factor. The bilinear form $a(\cdot, \cdot)$ is defined as

$$
a(\boldsymbol{\phi}, \boldsymbol{\psi})=\frac{E}{12\left(1-\nu^{2}\right)} \int_{\Omega}[(1-\nu) \mathcal{E}(\boldsymbol{\phi}): \mathcal{E}(\boldsymbol{\psi})+\nu \nabla \cdot \phi \nabla \cdot \boldsymbol{\psi}] d x d y,
$$

where $\mathcal{E}(\phi)=1 / 2\left[\nabla \phi+\nabla \phi^{T}\right]$ and $-1<\nu<1 / 2$. 
In our analysis we shall make use of a mixed formulation of the ReissnerMindlin plate equations proposed by Brezzi and Fortin in [4] based on the following Helmholtz decomposition of the shear force vector:

$$
\lambda t^{-2}(\nabla \omega-\phi)=\nabla r+\operatorname{curl} p
$$

with $(r, p) \in H_{0}^{1}(\Omega) \times \hat{H}^{1}(\Omega)$. With this decomposition, Problem 2.1 can be written as the following Brezzi-Fortin mixed formulation

Problem 2.2. Find $(r, \boldsymbol{\phi}, p, \omega) \in H_{0}^{1}(\Omega) \times\left(H_{0}^{1}(\Omega)\right)^{2} \times \hat{H}^{1}(\Omega) \times H_{0}^{1}(\Omega)$ such that

$$
\begin{gathered}
(\nabla r, \nabla \mu)=(g, \mu), \quad \forall \mu \in H_{0}^{1}(\Omega), \\
a(\phi, \boldsymbol{\psi})-(\operatorname{curl} p, \boldsymbol{\psi})=(\nabla r, \boldsymbol{\psi}), \quad \forall \boldsymbol{\psi} \in\left(H_{0}^{1}(\Omega)\right)^{2}, \\
-(\boldsymbol{\phi}, \operatorname{curl} q)-\lambda^{-1} t^{2}(\operatorname{curl} p, \operatorname{curl} q)=0, \quad \forall q \in \hat{H}^{1}(\Omega), \\
(\nabla \omega, \nabla s)=\left(\phi+\lambda^{-1} t^{2} \nabla r, \nabla s\right), \quad \forall s \in H_{0}^{1}(\Omega) .
\end{gathered}
$$

The following result concerning the existence and uniqueness of solutions to Problem 2.2 and the regularity can be found in 4,3 .

Lemma 2.3. Let $\Omega$ be a convex polygon or smoothly bounded domain in the plane. For any $t \in(0,1]$ and any $g \in H^{-1}(\Omega)$, there exists a unique quadruple $(r, \phi, p, \omega) \in$ $H_{0}^{1}(\Omega) \times\left(H_{0}^{1}(\Omega)\right)^{2} \times \hat{H}^{1}(\Omega) \times H_{0}^{1}(\Omega)$ solving Problem 2.2. Moreover, $\phi \in H^{2}(\Omega)$ and there exists a constant $C$ independent of $t$ and $g$, such that

$$
\|r\|_{1}+\|\phi\|_{2}+\|p\|_{1}+t\|p\|_{2}+\|\omega\|_{1} \leq C\|g\|_{-1} .
$$

If $g \in L^{2}(\Omega)$, then $r, \omega \in H^{2}(\Omega)$ and

$$
\|r\|_{2}+\|\omega\|_{2} \leq C\|g\|_{0} .
$$

\section{Finite Element method For the R-M Plate}

For approximating Problem 2.1 by the finite element method, we introduce a rectangular mesh $J^{h}$ of the rectangular domain $\Omega$. The regularity of the mesh $J^{h}$ is assumed in the sense of Ciarlet [12] such that $\bigcup_{K \in J^{h}} K=\bar{\Omega}$, the two distinct elements $K$ and $K^{\prime}$ in $J^{h}$ are either disjoint, or share the common edge $e$, or a common vertex. Let $F$ denote the set of all edges in $J^{h}$ with $F^{\prime}$ the set of interior edges. Given any edge $e \in F$ we assign a unit normal $\boldsymbol{n}_{e}$. In relation to $\boldsymbol{n}_{e}$ one can define the element $K^{+} \in J^{h}$ and the element $K^{-} \in J^{h}$, with $e=K^{+} \cap K^{-}$. Let $\partial K$ denote the boundary of $K$.

For each $K \in J^{h}$, we introduce the following affine invertible transformation:

$$
F_{K}: \hat{K} \rightarrow K, x=\frac{h_{x, K}}{2} \xi+x_{0, K}, \quad y=\frac{h_{y, K}}{2} \eta+y_{0, K}
$$

with $\left(x_{0, K}, y_{0, K}\right)$ the center and $h_{x, K}$ and $h_{y, K}$ the horizontal and vertical edge length of $K$, respectively, and $\hat{K}=[-1,1]^{2}$ the reference element. Let $Q_{1}(\hat{K})$ denote the usual bilinear function space on the reference elment $\hat{K}$, and set

$$
b(\xi, \eta)=(1+\xi+\eta)\left(1-\xi^{2}\right)\left(1-\eta^{2}\right) .
$$

Obviously, $b(\xi, \eta)$ is a bubble function and can be condensed out on the element level. 
Remark 3.1. The factor $1+\xi+\eta$ is added so that the space pair $\left(\boldsymbol{V}_{1, h}, Q_{h}\right)$ satisfies the discrete B-B condition; cf. Lemma 5.1 below. Using the bubble $\left(1-\xi^{2}\right)\left(1-\eta^{2}\right)$ instead, one can show by a similar argument of Lemma 5.1 that the space $N_{M}$ defined in Lemma 5.1 is not one-dimensional. This in turn implies that the discrete $\mathrm{B}-\mathrm{B}$ condition is not valid.

Define

$$
V_{h}^{c}:=\left\{v \in H_{0}^{1}(\Omega):\left.v\right|_{K} \circ F_{K} \in Q_{1}(\hat{K}) \oplus \operatorname{span}(b), \forall K \in J^{h}\right\} .
$$

Denote by $\mathcal{Q}_{1}(\hat{K})$ the modified nonconforming rotated $Q_{1}$ element space defined by

$$
\mathcal{Q}_{1}(\hat{K})=\operatorname{span}\left\{1, \xi, \eta, \xi^{2}-\eta^{2}, 1-\frac{3}{4}\left(\xi^{2}+\eta^{2}\right)\right\} .
$$

Note that the nonconforming bubble function $1-\frac{3}{4}\left(\xi^{2}+\eta^{2}\right)$ in (9) can also be condensed out on the element level with small effort.

For any $v \in H^{1}(K)$, define the following edge functional:

$$
\mathcal{F}_{e}(v)=\frac{1}{h_{e}} \int_{e} v d s
$$

with $e \subset \partial K$ and $h_{e}$ the length of the edge $e$. The modified nonconforming rotated $Q_{1}$ element space $V_{h}^{n c}$ is then defined as [13, 22, 15]

$$
\begin{aligned}
V_{h}^{n c}:= & \left\{v \in L^{2}(\Omega):\left.v\right|_{K} \circ F_{K} \in \mathcal{Q}_{1}(\hat{K}) \text { for each } K \in J^{h}, v\right. \text { continuous } \\
& \text { with respect to } \left.\mathcal{F}_{e} \text { for all } e \in F^{\prime}, \text { and } \mathcal{F}_{e}(v)=0 \text { for all } e \text { on } \partial \Omega\right\} .
\end{aligned}
$$

Define

$$
\boldsymbol{V}_{1, h}=V_{h}^{c} \times V_{h}^{c} \text { and } \boldsymbol{V}_{2, h}=V_{h}^{n c} \times V_{h}^{n c}
$$

as the approximation space of the rotation.

Define the discrete norm and semi-norm on $V_{h}^{n c}$ by

$$
\|v\|_{1, h}^{2}=\sum_{K \in J^{h}}\|v\|_{1, K}^{2}, \quad|v|_{1, h}^{2}=\sum_{K \in J^{h}}|v|_{1, K}^{2} .
$$

By Poincare's inequality [22], we have $|\cdot|_{1, h}$ as a norm on $V_{h}^{n c}$. The same rule is applicable to functions in $\boldsymbol{V}_{2, h}$.

To deal with the discontinuity of $\boldsymbol{V}_{2, h}$, we follow the idea in [11, 16, 18] and define for any vector-valued function $\boldsymbol{\psi} \in \boldsymbol{\Pi}_{K \in J^{h}} H^{1}(K)$ the jump across the edge $e \in F^{\prime}$ as

$$
[\boldsymbol{\psi}]=\left(\boldsymbol{\psi}^{+} \otimes \boldsymbol{n}^{+}\right)_{S}+\left(\boldsymbol{\psi}^{-} \otimes \boldsymbol{n}^{-}\right)_{S},
$$

where $(\boldsymbol{\psi} \otimes \boldsymbol{n})_{S}$ denotes the symmetric part of the tensor, and $\boldsymbol{n}^{+}\left(\right.$resp. $\left.\boldsymbol{n}^{-}\right)$is the outward unit normal to $e \subset \partial K^{+}$(resp. $e \subset \partial K^{-}$). On the boundary edge, we define the jump as $[\boldsymbol{\psi}]=(\boldsymbol{\psi} \otimes \boldsymbol{n})_{S}$ with $\boldsymbol{n}$ the outward unit normal to $\partial \Omega$.

Moreover, we introduce the following discrete bilinear form with a penalty term:

$$
\begin{aligned}
a_{h}\left(\boldsymbol{\phi}_{h}, \boldsymbol{\psi}_{h}\right)= & \frac{E}{12\left(1-\nu^{2}\right)} \sum_{K \in J^{h}} \int_{K}\left[(1-\nu) \mathcal{E}\left(\boldsymbol{\phi}_{h}\right): \mathcal{E}\left(\boldsymbol{\psi}_{h}\right)+\nu \nabla \cdot \boldsymbol{\phi}_{h} \nabla \cdot \boldsymbol{\psi}_{h}\right] d x d y \\
& +\sum_{e \in F} \frac{\gamma_{e}}{h_{e}} \int_{e}\left[\boldsymbol{\phi}_{h}\right]:\left[\boldsymbol{\psi}_{h}\right] d s
\end{aligned}
$$


with $\gamma_{e}$ some constant. For the analysis, we need to define the following auxiliary pressure finite element space and the discrete shear force space, respectively,

$$
\begin{gathered}
Q_{h}=\left\{q \in \hat{H}^{1}(\Omega):\left.q\right|_{K} \circ F_{K} \in Q_{1}(\hat{K}), \forall K \in J^{h}\right\}, \\
\boldsymbol{\Gamma}_{h}=\left\{\boldsymbol{v} \in\left(L^{2}(\Omega)\right)^{2}:\left.\boldsymbol{v}\right|_{K}=\left(\begin{array}{c}
b+d x \\
c+e y
\end{array}\right), \forall K \in J^{h}\right\} .
\end{gathered}
$$

Remark 3.2. The following shear force space is used in [28]:

$$
M=\left\{\boldsymbol{v} \in\left(L^{2}(\Omega)\right)^{2}:\left.\boldsymbol{v}\right|_{K}=\left(\begin{array}{c}
b+d x \\
c-d y
\end{array}\right), \forall K \in J^{h}\right\} .
$$

However, a close observation finds that $\nabla_{h} M_{1} \notin M$ (in the notation of [28]) for general rectangular meshes unless $h_{x, K}=h_{y, K}$, therefore the analysis therein is only valid for square meshes.

Let $\boldsymbol{R}_{h}:\left(L^{2}(\Omega)\right)^{2} \rightarrow \boldsymbol{\Gamma}_{h}$ denote the usual $L^{2}$ projection operator; then our finite element methods for the R-M plate problem can be stated as

Problem 3.3. Find $\left(\omega_{h}, \phi_{h}\right) \in V_{h}^{n c} \times \boldsymbol{V}_{i, h}$, such that

(11) $a_{h}\left(\boldsymbol{\phi}_{h}, \boldsymbol{\psi}\right)+\lambda t^{-2}\left(\nabla_{h} \omega_{h}-\boldsymbol{R}_{h} \boldsymbol{\phi}_{h}, \nabla_{h} v-\boldsymbol{\psi}\right)=(g, v), \quad \forall(v, \boldsymbol{\psi}) \in V_{h}^{n c} \times \boldsymbol{V}_{i, h}$, with $i=1,2$.

Remark 3.4. Notice that the penalty term in (10) vanishes for the space $\boldsymbol{V}_{1, h}$; we keep it there only for the convenience of the presentation and the simplicity of the notation.

\section{Discrete Helmholtz Decomposition}

\section{AND EQUIVALENT FORMULATIONS OF DISCRETE PROBLEMS}

In this section we prove the discrete Helmholtz Decomposition and present the equivalent formulation of the discrete problem.

Denote

$$
C_{h}=\left\{\boldsymbol{v} \mid \boldsymbol{v}=\operatorname{curl} q, q \in Q_{h}\right\} \text { and } G_{h}=\left\{\boldsymbol{v} \mid \boldsymbol{v}=\nabla_{h} w, w \in V_{h}^{n c}\right\} .
$$

Note that

$$
C_{h} \subset \boldsymbol{\Gamma}_{h} \text { and } G_{h} \subset \boldsymbol{\Gamma}_{h} \text {. }
$$

Furthermore, a counting argument gives

$$
\operatorname{dim} G_{h}+\operatorname{dim} C_{h}=\operatorname{dim} \boldsymbol{\Gamma}_{h} .
$$

Lemma 4.1. There holds

$$
C_{h} \perp G_{h} .
$$

Proof. Let $w \in V_{h}^{n c}$ and $q \in Q_{h}$, and

$$
\left(\nabla_{h} w, \operatorname{curl} q\right)=\sum_{K \in J^{h}} \int_{K} \nabla w \cdot \operatorname{curl} q d x d y=-\sum_{K \in J^{h}} \int_{\partial K} w \frac{\partial q}{\partial \boldsymbol{t}} d s,
$$

where $\boldsymbol{t}$ is the counterclockwise unit tangential vector to $\partial K$. Since $\frac{\partial q}{\partial t}$ is continuous constant on each edge of the element $K$, we have

$$
\left(\nabla_{h} w, \operatorname{curl} q\right)=-\sum_{e \in F^{\prime}} \int_{e}[w] \frac{\partial q}{\partial \boldsymbol{t}} d s-\sum_{e \in F \backslash F^{\prime}} \int_{e} w \frac{\partial q}{\partial \boldsymbol{t}} d s=0
$$

with $[w]$ the jump across $e$. 
Thanks to Lemma 4.1 and (13), we have the following discrete Helmholtz Decomposition:

$$
\Gamma_{h}=C_{h} \oplus G_{h}
$$

We now introduce the following auxiliary discrete problem

Problem 4.2. Find $\left(r_{h}, \phi_{h}, p_{h}, \omega_{h}\right) \in V_{h}^{n c} \times \boldsymbol{V}_{i, h} \times Q_{h} \times V_{h}^{n c}$ such that

$$
\begin{gathered}
\left(\nabla_{h} r_{h}, \nabla_{h} \mu\right)=(g, \mu), \quad \forall \mu \in V_{h}^{n c}, \\
a_{h}\left(\phi_{h}, \boldsymbol{\psi}\right)-\left(\operatorname{curl} p_{h}, \boldsymbol{\psi}\right)=\left(\nabla_{h} r_{h}, \boldsymbol{\psi}\right), \quad \forall \boldsymbol{\psi} \in \boldsymbol{V}_{i, h}, \\
-\left(\phi_{h}, \operatorname{curl} q\right)-\lambda^{-1} t^{2}\left(\operatorname{curl} p_{h}, \operatorname{curl} q\right)=0, \quad \forall q \in Q_{h}, \\
\left(\nabla_{h} \omega_{h}, \nabla_{h} s\right)=\left(\phi_{h}+\lambda^{-1} t^{2} \nabla_{h} r_{h}, \nabla_{h} s\right), \quad \forall s \in V_{h}^{n c},
\end{gathered}
$$

with $i=1,2$.

Theorem 4.3. For any $g \in L^{2}(\Omega)$ and $t \in(0,1]$ there exists a unique solution $\left(r_{h}, \phi_{h}, p_{h}, \omega_{h}\right)$ to Problem 4.2. Moreover, the pair $\left(\omega_{h}, \phi_{h}\right)$ is the unique solution of Problem 3.3 and

$$
\lambda t^{-2}\left(\nabla_{h} \omega_{h}-\boldsymbol{R}_{h} \phi_{h}\right)=\nabla_{h} r_{h}+\operatorname{curl} p_{h} .
$$

Proof. The existence and uniqueness of the solution to Problem 4.2 follows immediately from the discrete inf-sup condition (see Lemma 5.3 and Lemma 5.4 below) and the Korn inequality (see Lemma 5.5 below ) and Lemma 5.7 (see the next section for details).

Now we prove that $\left(\omega_{h}, \phi_{h}\right)$ is the unique solution to Problem 3.3 and that (19) holds. We use the orthogonality and definition of $\boldsymbol{R}_{h}$, (17) and (18) to get

$$
\begin{aligned}
\left(\nabla_{h} \omega_{h}-\boldsymbol{R}_{h} \boldsymbol{\phi}_{h}, \operatorname{curl} q\right) & =\lambda^{-1} t^{2}\left(\nabla_{h} r_{h}+\operatorname{curl} p_{h}, \operatorname{curl} q\right)=0, \quad \forall q \in Q_{h}, \\
\left(\nabla_{h} \omega_{h}-\boldsymbol{R}_{h} \boldsymbol{\phi}_{h}, \nabla_{h} s\right) & =\lambda^{-1} t^{2}\left(\nabla_{h} r_{h}+\operatorname{curl} p_{h}, \nabla_{h} s\right), \quad \forall s \in V_{h}^{n c},
\end{aligned}
$$

which imply that

$$
\nabla_{h} \omega_{h}-\boldsymbol{R}_{h} \boldsymbol{\phi}_{h}=\lambda^{-1} t^{2}\left(\nabla_{h} r_{h}+\operatorname{curl} p_{h}\right) .
$$

Thanks to (22), (16) and (18) can be written as, respectively

$$
\begin{gathered}
a_{h}\left(\boldsymbol{\phi}_{h}, \boldsymbol{\psi}\right)-\lambda t^{-2}\left(\nabla_{h} \omega_{h}-\boldsymbol{R}_{h} \boldsymbol{\phi}_{h}, \boldsymbol{\psi}\right)=0, \quad \forall \boldsymbol{\psi} \in \boldsymbol{V}_{i, h}, i=1,2, \\
\lambda t^{-2}\left(\boldsymbol{R}_{h} \boldsymbol{\phi}_{h}-\nabla_{h} \omega_{h}, \nabla_{h} s\right)=-\left(\nabla_{h} r_{h}, \nabla_{h} s\right), \quad \forall s \in V_{h}^{n c} .
\end{gathered}
$$

We obtain from (23) and (24) that

$$
a_{h}\left(\phi_{h}, \boldsymbol{\psi}\right)+\lambda t^{-2}\left(\nabla_{h} \omega_{h}-\boldsymbol{R}_{h} \boldsymbol{\phi}_{h}, \nabla_{h} s-\boldsymbol{\psi}\right)=\left(\nabla_{h} r_{h}, \nabla_{h} s\right) .
$$

By virtue of (15) and (25), we come to

$$
a_{h}\left(\phi_{h}, \boldsymbol{\psi}\right)+\lambda t^{-2}\left(\nabla_{h} \omega_{h}-\boldsymbol{R}_{h} \boldsymbol{\phi}_{h}, \nabla_{h} s-\boldsymbol{\psi}\right)=(g, s),
$$

which ends the proof. 


\section{The WeLL-POSEDNESS OF THE DISCRETE PROBLEMS}

In this section we shall show the well-posedness of the discrete Problem 4.2 . Because (15) and (18) are elliptic problems which are decoupled from the system, and their well-posedness follows immediately from Lemma 5.7, we only need to show the well-posedness for Stokes-like problem (16)-(17), which hangs on the discrete inf-sup condition, namely the B-B condition and the continuity and coercivity of $a_{h}$. We first prove the discrete inf-sup condition for the pairs $\left(\boldsymbol{V}_{i, h}, Q_{h}\right), i=1,2$. To this end, we shall use the macroelement trick from [26, 9].

For any interior node $P_{i}$, we define the associated macroelement by

$$
M\left(P_{i}\right)=\left\{K \mid K \cap P_{i} \neq \emptyset, K \in J^{h}\right\} .
$$

Lemma 5.1. There exists a positive constant $\beta$ independent of $h$, such that

$$
\sup _{\boldsymbol{\psi} \in \boldsymbol{V}_{1, h}} \frac{(\operatorname{div} \boldsymbol{\psi}, q)}{\|\boldsymbol{\psi}\|_{1}} \geq \beta\|q\|_{0}, \quad \forall q \in Q_{h} .
$$

Proof. Let $M$ be a macroelement with nodes $P_{i}\left(x_{i}, y_{i}\right), i=1, \cdots, 9$, and elements $K_{i}, i=1, \cdots, 4$ (see Figure 11). Define

$$
\begin{gathered}
S_{2, M}:=\left\{\boldsymbol{\psi}: \boldsymbol{\psi} \in\left(H_{0}^{1}(M)\right)^{2} \cap \boldsymbol{V}_{1, h}\right\}, \\
S_{3, M}=\left\{q: q \in H^{1}(M) \cap Q_{h}\right\}, \\
N_{M}=\left\{q \in S_{3, M}:(\operatorname{div} \boldsymbol{\psi}, q)=0, \forall \boldsymbol{\psi} \in S_{2, M}\right\} .
\end{gathered}
$$

By a theory from [26], we only need to show that $N_{M}$ is one-dimensional.

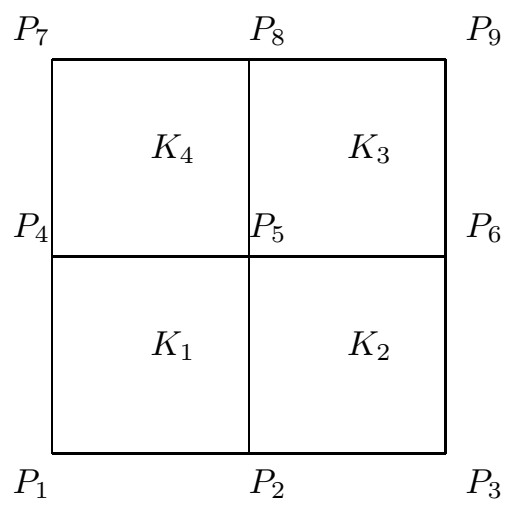

Figure 1. Macroelement

Denote the bilinear basis on nodes $P_{1}, \cdots, P_{9}$ by $\phi_{1}, \cdots, \phi_{9}$, and the bubble basis function of $V_{h}^{c}$ on the element $K_{1}, \cdots, K_{4}$ by $\varphi_{1}, \cdots, \varphi_{4}$ respectively. We have for any $\boldsymbol{\psi} \in S_{2, M}$ and $q \in S_{3, M}$ the following expressions:

$$
\psi=\sum_{i=1}^{i=4}\left(\begin{array}{l}
v_{1, i} \\
v_{2, i}
\end{array}\right) \varphi_{i}+\left(\begin{array}{c}
v_{1,5} \\
v_{2,5}
\end{array}\right) \phi_{5} \text { and } q=\sum_{i=1}^{9} a_{i} \phi_{i}
$$

Integrating by parts, we have

$$
(\operatorname{div} \boldsymbol{\psi}, q)=-(\boldsymbol{\psi}, \nabla q)=-\sum_{i=1}^{4}(\boldsymbol{\psi}, \nabla q)_{K_{i}}
$$


for any $\boldsymbol{\psi} \in S_{2, M}$ and $q \in S_{3, M}$. Take $v_{2, i}=0, i=1, \cdots, 4, v_{1,5}=v_{2,5}=0$, $v_{1, i}=0, i=2,3,4$, and $v_{1,1}=1$ and set

$$
0=(\operatorname{div} \boldsymbol{\psi}, q)=-(\boldsymbol{\psi}, \nabla q) .
$$

This yields

$$
\begin{aligned}
0= & \int_{K_{1}} \varphi_{1} \frac{\partial\left(a_{1} \phi_{1}+a_{2} \phi_{2}+a_{4} \phi_{4}+a_{5} \phi_{5}\right)}{\partial x} d x d y \\
= & \frac{h_{y, K_{1}}}{2} \int_{\hat{K}}(1+\xi+\eta)\left(1-\xi^{2}\right)\left(1-\eta^{2}\right)\left(-a_{1}+a_{2}-a_{4}+a_{5}\right) d \xi d \eta \\
& +\frac{h_{y, K_{1}}}{2} \int_{\hat{K}}(1+\xi+\eta)\left(1-\xi^{2}\right)\left(1-\eta^{2}\right)\left(a_{1}-a_{2}-a_{4}+a_{5}\right) \eta d \xi d \eta .
\end{aligned}
$$

Then a direct calculation gives

$$
2 a_{1}-2 a_{2}+3 a_{4}-3 a_{5}=0 .
$$

Similarly, let $v_{2, i}=0$ with $i=2, \cdots, 4, v_{1,5}=v_{2,5}=0, v_{1, i}=0, i=1,2,3,4$, and $v_{2,1}=1$. We have

$$
-2 a_{1}-3 a_{2}+2 a_{4}+3 a_{5}=0 .
$$

Now let one of degrees $v_{j, i}, i=2,3,4, j=1,2$, be 1 and the others be zero successively. We can get a system of equations with respect to $\left(a_{1}, \cdots, a_{9}\right)$, which reads

$$
\left\{\begin{array}{l}
2 a_{1}-2 a_{2}+3 a_{4}-3 a_{5}=0 \\
-2 a_{1}-3 a_{2}+2 a_{4}+3 a_{5}=0 \\
2 a_{2}-2 a_{3}+3 a_{5}-3 a_{6}=0 \\
-2 a_{2}-3 a_{3}+2 a_{5}+3 a_{6}=0 \\
2 a_{4}-2 a_{5}+3 a_{7}-3 a_{8}=0 \\
-2 a_{4}-3 a_{5}+2 a_{7}+3 a_{8}=0 \\
2 a_{5}-2 a_{6}+3 a_{8}-3 a_{9}=0 \\
-2 a_{5}-3 a_{6}+2 a_{8}+3 a_{9}=0
\end{array}\right.
$$

We solve this system to get

$$
\left\{\begin{array}{lll}
a_{1}=\frac{13 a-9 b}{4} & a_{2}=\frac{9 b-7 a}{2} & a_{3}=a, \\
a_{4}=\frac{9 b-7 a}{2} & a_{5}=a & a_{6}=3 b-2 a, \\
a_{7}=a & a_{8}=3 b-2 a & a_{9}=b,
\end{array}\right.
$$

such that $a$ and $b$ are two free parameters. Finally we take $v_{2,5}=1$, and $v_{1, i}=0$, $i=1, \cdots, 4, v_{2, i}=0, i=1, \cdots, 4, v_{1,5}=0$, to obtain $a=b$; therefore

$$
a_{1}=\cdots=a_{9} .
$$

This is to say $N_{M}$ is one dimensional.

Remark 5.2. Note from the above calculation that we can only obtain the following system:

$$
\left\{\begin{array}{l}
-a_{1}+a_{2}-a_{4}+a_{5}=0 \\
-a_{1}-a_{2}+a_{4}+a_{5}=0 \\
-a_{2}+a_{3}-a_{5}+a_{6}=0 \\
-a_{2}-a_{3}+a_{5}+a_{6}=0 \\
-a_{4}+a_{5}-a_{7}+a_{8}=0 \\
-a_{4}-a_{5}+a_{7}+a_{8}=0 \\
-a_{5}+a_{6}-a_{8}+a_{9}=0 \\
-a_{5}-a_{6}+a_{8}+a_{9}=0
\end{array}\right.
$$


if we employ $\left(1-\xi^{2}\right)\left(1-\eta^{2}\right)$ instead. This leads to

$$
a_{1}=a_{3}=a_{5}=a_{7}=a_{9}, \quad a_{2}=a_{4}=a_{6}=a_{7}=a_{8} .
$$

With these identities, we cannot prove $a_{1}=a_{2}$ by taking $v_{1,5}=1$ or $v_{2,5}=1$, and letting the remaining degrees of freedom be zero. Therefore $N_{M}$ is two-dimensional in this case.

Lemma 5.3. There holds that

$$
\sup _{\boldsymbol{\psi} \in \boldsymbol{V}_{1, h}} \frac{(\operatorname{rot} \phi, q)}{\|\phi\|_{1}}=\sup _{\boldsymbol{\psi} \in \boldsymbol{V}_{1, h}} \frac{(\boldsymbol{\phi}, \operatorname{curl} q)}{\|\phi\|_{1}} \geq \beta\|q\|_{0}, \quad \forall q \in Q_{h} .
$$

Proof. Let $\psi_{2}=-\phi_{1}$ and $\psi_{1}=\phi_{2}$ in Lemma 5.1. We obtain with $\phi=\left(\phi_{1}, \phi_{2}\right)$ and $\boldsymbol{\psi}=\left(\psi_{1}, \psi_{2}\right)$

$$
\begin{aligned}
\sup _{\phi \in V_{1, h}} \frac{(\operatorname{rot} \phi, q)}{\|\phi\|_{1}} & =\sup _{\phi \in V_{1, h}} \frac{(\phi, \operatorname{curl} q)}{\|\phi\|_{1}} \\
& =\sup _{\boldsymbol{\psi} \in V_{1, h}} \frac{(\operatorname{div} \boldsymbol{\psi}, q)}{\|\boldsymbol{\psi}\|_{1}} \geq \beta\|q\|_{0} \quad \forall q \in Q_{h} .
\end{aligned}
$$

This ends the proof.

By the same argument of Lemma 5.1, we have

Lemma 5.4. There exists a positive constant $\beta$ such that

$$
\sup _{\boldsymbol{\psi} \in \boldsymbol{V}_{2, h}} \frac{(\boldsymbol{\psi}, \operatorname{curl} q)}{\|\boldsymbol{\psi}\|_{1, h}} \geq \beta\|q\|_{0}, \quad \forall q \in Q_{h} .
$$

To prove the well-posedness of the discrete problem, we remain to show the continuity and coercivity of $a_{h}$, i.e.,

Lemma 5.5. There exist two positive constants $C_{1}$ and $C_{2}$ independent of $h$ and $t$ such that

$$
\begin{gathered}
C_{1}\|\boldsymbol{\psi}\|_{1, h}^{2} \leq a_{h}(\boldsymbol{\psi}, \boldsymbol{\psi}), \quad \forall \boldsymbol{\psi} \in \boldsymbol{V}_{i, h}, i=1,2, \\
\left|a_{h}(\boldsymbol{\phi}, \boldsymbol{\psi})\right| \leq C_{2}\|\boldsymbol{\phi}\|_{1, h}\|\boldsymbol{\psi}\|_{1, h}, \quad \forall \boldsymbol{\phi}, \boldsymbol{\psi} \in \boldsymbol{V}_{i, h}, i=1,2 .
\end{gathered}
$$

Proof. Lemma 5.5 holds obviously for the space $\boldsymbol{V}_{1, h}$, and it is also easy to show (32) for $\boldsymbol{V}_{2, h}$. The proof of (31) for $\boldsymbol{V}_{2, h}$ follows immediately from Lemma 5.6 and Lemma 5.7 below.

Lemma 5.6. There exists a positive constant $C$ independent of $h$ such that

$$
C|\boldsymbol{\psi}|_{1, h} \leq\left\|\mathcal{E}_{h}(\boldsymbol{\psi})\right\|_{0}+\left(\sum_{e \in F} \frac{1}{h_{e}} \int_{e}[\boldsymbol{\psi}]^{2} d s\right)^{1 / 2}, \quad \forall \boldsymbol{\psi} \in \boldsymbol{V}_{2, h}
$$

Proof. The proof can be found, for instance, in $[8]$.

Lemma 5.7. There exists a positive constant $C$ independent of $h$ such that

$$
C\|v\|_{0} \leq|v|_{1, h}, \quad \forall v \in V_{h}^{n c} .
$$

Proof. The proof can be found, for instance, in [7, 22, 15]. 


\section{ERror estimate}

We show error estimates in this section. We first derive some approximation results. For the modified nonconforming rotated $Q_{1}$ element, we define the interpolation operator $\pi_{h}: H_{0}^{1}(n) \rightarrow V_{h}^{n c}$ by

$$
\begin{aligned}
& \int_{e} \pi_{h} v d s=\int_{e} v d s, \quad \forall v \in H_{0}^{1}(n), \text { for any } e \in F, \\
& \int_{K} \pi_{h} v d x d y=\int_{K} v d x d y, \text { for any } K \in J^{h} .
\end{aligned}
$$

We have the following result

\section{Lemma 6.1.}

$$
\begin{aligned}
& \left|\sum_{K} \int_{\partial K} v \psi \cdot n d s\right| \leq C h\|v\|_{1, h}\|\psi\|_{1}, \quad \forall v \in V_{h}^{n c}, \quad \forall \psi \in\left(H^{1}(\Omega)\right)^{2}, \\
& \left\|v-\pi_{h} v\right\|+h\left|v-\pi_{h} v\right|_{1, h} \leq C h^{2}|v|_{2}, \quad \forall v \in H^{2}(n) \cap H_{0}^{1}(n),
\end{aligned}
$$

Proof. The proof can be found in [13, 22, 15].

Remark 6.2. Note that (36) is obviously satisfied by $V_{h}^{c} \in H_{0}^{1}(\Omega)$, consequently we shall not differ $\boldsymbol{V}_{1, h}$ from $\boldsymbol{V}_{2, h}$ when the consistency error is concerned.

For the projection operator $\boldsymbol{R}_{h}$, we have the following approximation property,

Lemma 6.3. There exists a constant $C$, for any $\boldsymbol{u} \in\left(H^{1}(\Omega)\right)^{2}$, such that

$$
\left\|\boldsymbol{R}_{h} \boldsymbol{u}-\boldsymbol{u}\right\|_{0} \leq C h\|\boldsymbol{u}\|_{1} .
$$

Proof. The proof is elementary.

Lemma 6.4. Let $G \in L^{2}(\Omega)$ and $F \in\left(H^{1}(\Omega)\right)^{2}$, $u$ be the weak solution to the following boundary value problem

$$
\begin{gathered}
-\triangle u=G-\nabla \cdot F \text { in } \Omega, \\
\left.u\right|_{\partial \Omega}=0,
\end{gathered}
$$

and $u_{h} \in V_{h}^{n c}$ be the solution to the discrete problem

$$
\left(\nabla_{h} u_{h}, \nabla_{h} v\right)=(G, v)+\left(F, \nabla_{h} v\right), \quad \forall v \in V_{h}^{n c} .
$$

Then there exists a constant $C$ independent of $h, G$ and $F$ such that

$$
\begin{gathered}
\left\|u-u_{h}\right\|_{1, h} \leq C h\left(\|G\|_{0}+\|F\|_{1}\right), \\
\left\|u-u_{h}\right\|_{0} \leq C h^{2}\left(\|G\|_{0}+\|F\|_{1}\right) .
\end{gathered}
$$

Proof. Using Lemma 6.1, we can obtain (42)-(43) by standard arguments from nonconforming finite element methods for the second order elliptic problems 24, 25, 6]. For the brevity, we omit the details.

Theorem 6.5. Let $(r, \phi, p, \omega)$ and $\left(r_{h}, \phi_{h}, p_{h}, \omega_{h}\right)$ be the solution to Problem 2.2 and 4.2, respectively. For any $g \in L^{2}(\Omega)$ and $t \in(0,1]$, there exists a constant $C$ independent of $h, g$ and $t$, such that

$$
\begin{aligned}
& \left\|r-r_{h}\right\|_{1, h}+\left\|\phi-\phi_{h}\right\|_{1, h}+\left\|p-p_{h}\right\|_{0}+t\left\|\operatorname{curl}\left(p-p_{h}\right)\right\|_{0} \\
& \quad+\left\|\omega-\omega_{h}\right\|_{1, h} \leq C h\|g\|_{0} .
\end{aligned}
$$


Proof. Throughout the proof, $i=1,2$. Owing to (3), (15) and Lemma 6.4, we have

$$
\left\|\nabla_{h}\left(r-r_{h}\right)\right\|_{0} \leq C h\|g\|_{0} .
$$

We have by (4) and (16) for any $\boldsymbol{\psi} \in \boldsymbol{V}_{i, h}, i=1,2$, that

$$
\begin{aligned}
a_{h}\left(\boldsymbol{\phi}_{h}-\boldsymbol{\psi}, \boldsymbol{\phi}_{h}-\boldsymbol{\psi}\right) & =a_{h}\left(\boldsymbol{\phi}-\boldsymbol{\psi}, \boldsymbol{\phi}_{h}-\boldsymbol{\psi}\right) \\
& +\left(\operatorname{curl}\left(p_{h}-p\right), \boldsymbol{\phi}_{h}-\boldsymbol{\psi}\right)+\left(\nabla_{h} r_{h}-\nabla r, \boldsymbol{\phi}_{h}-\boldsymbol{\psi}\right) \\
& -a_{h}\left(\boldsymbol{\phi}, \boldsymbol{\phi}_{h}-\boldsymbol{\psi}\right)+\left(\operatorname{curl} p, \boldsymbol{\phi}_{h}-\boldsymbol{\psi}\right)+\left(\nabla r, \boldsymbol{\phi}_{h}-\boldsymbol{\psi}\right) .
\end{aligned}
$$

By (5) and (17), we obtain for any $\boldsymbol{\psi} \in \boldsymbol{V}_{i, h}$ and $q \in Q_{h}$ that

$$
\begin{aligned}
\lambda^{-1} t^{2}\left\|\operatorname{curl}\left(p_{h}-q\right)\right\|_{0}^{2}= & \lambda^{-1} t^{2}\left(\operatorname{curl}(p-q), \operatorname{curl}\left(p_{h}-q\right)\right)-\left(\phi_{h}-\boldsymbol{\phi}, \operatorname{curl}\left(p_{h}-q\right)\right) \\
= & \lambda^{-1} t^{2}\left(\operatorname{curl}(p-q), \operatorname{curl}\left(p_{h}-q\right)\right)-\left(\boldsymbol{\phi}_{h}-\boldsymbol{\psi}, \operatorname{curl}\left(p_{h}-q\right)\right) \\
& +\left(\boldsymbol{\phi}-\boldsymbol{\psi}, \operatorname{curl}\left(p_{h}-q\right)\right) .
\end{aligned}
$$

Taking these two equations together,

$$
\begin{aligned}
a_{h}\left(\phi_{h}-\boldsymbol{\psi}, \boldsymbol{\phi}_{h}-\boldsymbol{\psi}\right) & +\lambda^{-1} t^{2}\left\|\operatorname{curl}\left(p_{h}-q\right)\right\|_{0}^{2} \\
= & a_{h}\left(\boldsymbol{\phi}-\boldsymbol{\psi}, \boldsymbol{\phi}_{h}-\boldsymbol{\psi}\right) \\
& +\left(\nabla_{h} r_{h}-\nabla r, \boldsymbol{\phi}_{h}-\boldsymbol{\psi}\right)+\lambda^{-1} t^{2}\left(\operatorname{curl}(p-q), \operatorname{curl}\left(p_{h}-q\right)\right) \\
& -\left(\operatorname{curl}(p-q), \boldsymbol{\phi}_{h}-\boldsymbol{\psi}\right)+\left(\boldsymbol{\phi}-\boldsymbol{\psi}, \operatorname{curl}\left(p_{h}-q\right)\right) \\
& -a_{h}\left(\boldsymbol{\phi}, \boldsymbol{\phi}_{h}-\boldsymbol{\psi}\right)+\left(\operatorname{curl} p, \boldsymbol{\phi}_{h}-\boldsymbol{\psi}\right)+\left(\nabla r, \boldsymbol{\phi}_{h}-\boldsymbol{\psi}\right) .
\end{aligned}
$$

It follows from (36) (resp. Remark 6.2) and (4) that

$$
\left|a_{h}\left(\boldsymbol{\phi}, \boldsymbol{\phi}_{h}-\boldsymbol{\psi}\right)+\left(\operatorname{curl} p, \boldsymbol{\phi}_{h}-\boldsymbol{\psi}\right)+\left(\nabla r, \boldsymbol{\phi}_{h}-\boldsymbol{\psi}\right)\right| \leq C h\|\boldsymbol{\phi}\|_{2}\left|\boldsymbol{\phi}_{h}-\boldsymbol{\psi}\right|_{1, h} .
$$

For any $\boldsymbol{\psi} \in\left(H_{0}^{1}(n)\right)^{2} \cup \boldsymbol{V}_{i, h}$ and $q \in H^{1}(\Omega)$, we need to bound the term $(\boldsymbol{\psi}, \operatorname{curl} q)$. Integrating by parts and using (36), we derive it as

$$
\begin{aligned}
(\boldsymbol{\psi}, \operatorname{curl} q) & =-\sum_{K \in J^{h}} \int_{K} \operatorname{rot} \boldsymbol{\psi} q d x+\sum_{e \in E} \int_{e}[\boldsymbol{\psi}] \cdot \boldsymbol{t} q d s \\
& \leq C\left(\|\boldsymbol{\psi}\|_{1, h}\|q\|_{0}+h\|\boldsymbol{\psi}\|_{1, h}\|q\|_{1}\right) .
\end{aligned}
$$

Thanks to Lemma 5.3. Lemma 5.4 and Lemma 5.5, we get by (44), (16), (46) and (47) that

$$
\begin{aligned}
\beta\left\|p_{h}-q\right\|_{0} & \leq \sup _{\boldsymbol{\psi} \in \boldsymbol{V}_{i, h}} \frac{\left(\boldsymbol{\psi}, \operatorname{curl}\left(p_{h}-q\right)\right)}{\|\boldsymbol{\psi}\|_{1, h}} \\
& \leq C\left(\left\|\boldsymbol{\phi}_{h}-\boldsymbol{\phi}\right\|_{1, h}+\left\|\nabla_{h} r_{h}-\nabla r\right\|_{0}+h\|\boldsymbol{\phi}\|_{2}\right)+\sup _{\boldsymbol{\psi} \in \boldsymbol{V}_{i, h}} \frac{(\boldsymbol{\psi}, \operatorname{curl}(p-q))}{\|\boldsymbol{\psi}\|_{1, h}} \\
& \leq C\left(\left\|\boldsymbol{\phi}_{h}-\boldsymbol{\psi}\right\|_{1, h}+\|\boldsymbol{\phi}-\boldsymbol{\psi}\|_{1, h}+\left\|\nabla_{h} r_{h}-\nabla r\right\|_{0}+h\|\boldsymbol{\phi}\|_{2}\right) \\
& +C\left(h\|p-q\|_{1}+\|p-q\|_{0}\right) .
\end{aligned}
$$

Substituting (46), (48) and (47) into (45), and using Lemma 5.5, Lemma 5.7 and the inverse estimate, we proceed as

$$
\begin{aligned}
\left\|\phi_{h}-\boldsymbol{\psi}\right\|_{1, h} & +t\left\|\operatorname{curl}\left(p_{h}-q\right)\right\|_{0} \\
\leq & C\left(\|\boldsymbol{\phi}-\boldsymbol{\psi}\|_{1, h}+t\|p-q\|_{1}+\left\|\nabla_{h} r_{h}-\nabla r\right\|_{0}+h\|\boldsymbol{\phi}\|_{2}\right) \\
& +C\left(h\|p-q\|_{1}+\|p-q\|_{0}\right) .
\end{aligned}
$$


We now use the triangle inequality to obtain

$$
\begin{aligned}
\left\|\phi_{h}-\boldsymbol{\phi}\right\|_{1, h} & +t\left\|\operatorname{curl}\left(p_{h}-p\right)\right\|_{0} \\
& \leq C\left(\|\boldsymbol{\phi}-\boldsymbol{\psi}\|_{1, h}+t\|p-q\|_{1}+h\|p-q\|_{1}\right. \\
& \left.+\|p-q\|_{0}+\left\|\nabla_{h} r_{h}-\nabla r\right\|_{0}+h\|\phi\|_{2}\right) .
\end{aligned}
$$

It follows from (48) and (49) that

$$
\begin{aligned}
\left\|p_{h}-p\right\|_{0} \leq & C\left(\|\boldsymbol{\phi}-\boldsymbol{\psi}\|_{1, h}+t\|p-q\|_{1}+h\|p-q\|_{1}\right. \\
& \left.+\|p-q\|_{0}+\left\|\nabla_{h} r_{h}-\nabla r\right\|_{0}+h\|\boldsymbol{\phi}\|_{2}\right) .
\end{aligned}
$$

We take $q=\pi_{1}^{h} p$ with $\pi_{1}^{h}$ the bilinear Clement interpolation operator [23] which admits the following approximation property:

$$
\left\|p-\pi_{1}^{h} p\right\|_{0}+h\left\|p-\pi_{1}^{h} p\right\|_{1} \leq C h\|p\|_{1} \text { and }\left\|p-\pi_{1}^{h} p\right\|_{1} \leq C h\|p\|_{2} .
$$

Applying Lemma 2.3. Lemma 6.3 and Lemma 6.4, we finally obtain

$$
\|\boldsymbol{\phi}-\boldsymbol{\phi}\|_{1, h}+\left\|p-p_{h}\right\|_{0}+t\left\|\operatorname{curl}\left(p_{h}-p\right)\right\|_{0} \leq C h\|g\|_{0},
$$

since $\boldsymbol{\psi} \in \boldsymbol{V}_{i, h}$ is arbitrary.

Now, we remain to bound $\left\|\omega-\omega_{h}\right\|_{1, h}$. Let $\bar{\omega}_{h} \in V_{h}^{n c}$ be the solution to the following problem:

$$
\left(\nabla_{h} \bar{\omega}_{h}, \nabla_{h} s\right)=\left(\phi+\lambda^{-1} t^{2} \nabla r, \nabla_{h} s\right), \quad \forall s \in V_{h}^{n c} .
$$

Taking into account Lemma 6.4, we deduce

$$
\left\|\nabla_{h}\left(\omega-\bar{\omega}_{h}\right)\right\|_{0} \leq C h\left\|\phi+\lambda^{-1} t^{2} \nabla r\right\|_{1} \leq C h\|g\|_{0} .
$$

It follows from (6) and (18) that

$$
\left(\nabla_{h}\left(\omega_{h}-\bar{\omega}_{h}\right), \nabla_{h} s\right)=\left(\phi_{h}-\phi+\lambda^{-1} t^{2}\left(\nabla_{h} r_{h}-\nabla r\right), \nabla_{h} s\right) .
$$

Let $s=\omega_{h}-\bar{\omega}_{h}$ in (53); we have

$$
\left\|\nabla_{h}\left(\omega_{h}-\bar{\omega}_{h}\right)\right\|_{0} \leq C\left(\left\|\phi_{h}-\phi\right\|_{0}+t^{2}\left\|\nabla_{h} r_{h}-\nabla r\right\|_{0}\right) \leq C h\|g\|_{0},
$$

which, together with (52), implies

$$
\left\|\omega_{h}-\omega\right\|_{1, h} \leq C h\|g\|_{0},
$$

which completes the proof.

In order to analyse the $L^{2}$ error, we need to introduce the following dual problem

Problem 6.6. Find $\left(\phi_{d}, p_{d}\right) \in\left(H_{0}^{1}(n)\right)^{2} \times \hat{H}^{1}(\Omega)$, such that

$$
\begin{gathered}
a\left(\boldsymbol{\phi}_{d}, \boldsymbol{\psi}\right)-\left(\boldsymbol{\psi}, \operatorname{curl} p_{d}\right)=(d, \boldsymbol{\psi}), \quad \forall \boldsymbol{\psi} \in\left(H_{0}^{1}(\Omega)\right)^{2}, \\
-\left(\boldsymbol{\phi}_{d}, \operatorname{curl} q\right)-\lambda^{-1} t^{2}\left(\operatorname{curl} p_{d}, \operatorname{curl} q\right)=0, \quad \forall q \in \hat{H}^{1}(\Omega) .
\end{gathered}
$$

The solution to Problem 6.6 admits the following regularity:

$$
\left\|\phi_{d}\right\|_{2}+\left\|p_{d}\right\|_{1}+t\left\|p_{d}\right\|_{2} \leq C\|d\|_{0} .
$$

Define the following interpolation:

$$
\Pi_{h} \boldsymbol{\psi}=\left\{\begin{array}{l}
\left(\pi_{1}^{h} \psi_{1}, \pi_{1}^{h} \psi_{2}\right) \text { when } \boldsymbol{V}_{1, h} \text { is used, } \\
\left(\pi_{h} \psi_{1}, \pi_{h} \psi_{2}\right) \text { when } \boldsymbol{V}_{2, h} \text { is used }
\end{array}\right.
$$

for any $\left(\psi_{1}, \psi_{2}\right)=\boldsymbol{\psi} \in\left(H_{0}^{1}(\Omega)\right)^{2}$. We have the following estimates

$$
\left\|\Pi_{h} \boldsymbol{\psi}-\boldsymbol{\psi}\right\|_{0}+h\left\|\Pi_{h} \boldsymbol{\psi}-\boldsymbol{\psi}\right\|_{1, h} \leq C h\|\boldsymbol{\psi}\|_{1} \text { and }\left\|\Pi_{h} \boldsymbol{\psi}-\boldsymbol{\psi}\right\|_{1, h} \leq C h\|\boldsymbol{\psi}\|_{2} .
$$


Theorem 6.7. Let $(r, \phi, p, \omega)$ and $\left(r_{h}, \phi_{h}, p_{h}, \omega_{h}\right)$ be the solution to Problem 2.2 and 4.2, respectively. For any $g \in L^{2}(\Omega)$ and $t \in(0,1]$, there exists a constant $C$ independent of $h, g$ and $t$, such that

$$
\left\|\phi-\phi_{h}\right\|_{0}+\left\|\omega_{h}-\omega\right\|_{0} \leq C h^{2}\|g\|_{0} .
$$

Proof. First, it follows from Lemma 6.4 that

$$
\left\|r-r_{h}\right\|_{0} \leq C h^{2}\|g\|_{0} .
$$

Applying (16) and (17) as well as (54) and (55), we derive it as

$$
\begin{aligned}
& \left(d, \phi-\phi_{h}\right)=\left(d, \phi-\phi_{h}\right)-a_{h}\left(\phi_{d}, \phi-\phi_{h}\right)+\left(\phi-\phi_{h}, \operatorname{curl} p_{d}\right) \\
& +a\left(\phi_{d}, \phi-\phi_{h}\right)-\left(\phi-\phi_{h}, \operatorname{curl} p_{d}\right) \\
& =\left(d, \phi-\phi_{h}\right)-a_{h}\left(\phi_{d}, \boldsymbol{\phi}-\phi_{h}\right)+\left(\phi-\phi_{h}, \operatorname{curl} p_{d}\right) \\
& +a_{h}\left(\Pi_{h} \phi_{d}-\phi_{d}, \phi\right)-\left(\Pi_{h} \phi_{d}-\phi_{d}, \operatorname{curl} p\right)-\left(\nabla r, \Pi_{h} \phi_{d}-\phi_{d}\right) \\
& +a_{h}\left(\phi_{d}-\Pi_{h} \phi_{d}, \phi-\phi_{h}\right) \\
& -\left(\phi-\phi_{h}, \operatorname{curl}\left(p_{d}-\pi_{1}^{h} p_{d}\right)\right) \\
& +\left(\Pi_{h} \phi_{d}-\phi_{d}, \operatorname{curl}\left(p-p_{h}\right)\right) \\
& +\left(\nabla r-\nabla_{h} r_{h}, \Pi_{h} \phi_{d}\right) \\
& +\lambda^{-1} t^{2}\left(\operatorname{curl}\left(p-p_{h}\right), \operatorname{curl}\left(\pi_{1}^{h} p_{d}-p_{d}\right)\right)=I_{1}+\cdots+I_{7} .
\end{aligned}
$$

$I_{1}$ and $I_{2}$ are consistency error terms, which can be estimated by a classic argument

$$
\left|I_{1}\right| \leq C h\left\|\phi_{d}\right\|_{2}\left\|\phi-\phi_{h}\right\|_{1, h} \text { and }\left|I_{2}\right| \leq C h\|\phi\|_{2}\left\|\Pi_{h} \phi_{d}-\phi_{d}\right\|_{1, h} .
$$

Owing to Lemma 5.5

$$
\left|I_{3}\right| \leq C\left\|\Pi_{h} \phi_{d}-\phi_{d}\right\|_{1, h}\left\|\phi-\phi_{h}\right\|_{1, h} .
$$

Thanks to (47),

$$
\left|I_{4}\right| \leq C\left(\left\|\phi-\phi_{h}\right\|_{1, h}\left\|p_{d}-\pi_{1}^{h} p_{d}\right\|_{0}+h\left\|\phi-\phi_{h}\right\|_{1, h}\left\|p_{d}-\pi_{1}^{h} p_{d}\right\|_{1}\right) .
$$

Using (47) again, we have by the inverse and triangle inequality

$$
\begin{aligned}
I_{5} & =\left(\Pi_{h} \phi_{d}-\phi_{d}, \operatorname{curl}\left(p-\pi_{1}^{h} p\right)\right)+\left(\Pi_{h} \phi_{d}-\phi_{d}, \operatorname{curl}\left(\pi_{1}^{h} p-p_{h}\right)\right) \\
& \leq C\left\|\Pi_{h} \phi_{d}-\phi_{d}\right\|_{1, h}\left(\left\|p-\pi_{1}^{h} p\right\|_{0}+\left\|\pi_{1}^{h} p-p_{h}\right\|_{0}+h\left\|p-\pi_{1}^{h} p\right\|_{1}\right) \\
& \leq C\left\|\Pi_{h} \phi_{d}-\phi_{d}\right\|_{1, h}\left(\left\|p-\pi_{1}^{h} p\right\|_{0}+h\left\|p-\pi_{1}^{h} p\right\|_{1}+\left\|p-p_{h}\right\|_{0}\right) .
\end{aligned}
$$

We have the following decomposition for the sixth term $I_{6}$ :

$$
I_{6}=\left(\nabla r-\nabla_{h} r_{h}, \Pi_{h} \phi_{d}-\phi_{d}\right)+\left(\nabla r-\nabla_{h} r_{h}, \phi_{d}\right) .
$$

By virtue of Theorem 6.5. we bound the first term in (65) as

$$
\left(\nabla r-\nabla_{h} r_{h}, \Pi_{h} \phi_{d}-\phi_{d}\right) \leq C h\|g\|_{0}\left\|\Pi_{h} \phi_{d}-\phi_{d}\right\|_{1, h} .
$$

Integrating by parts and applying (59), the second term in (65) can be bounded as

$$
\begin{aligned}
\left(\nabla r-\nabla_{h} r_{h}, \phi_{d}\right) & =-\left(r-r_{h}, \operatorname{div} \boldsymbol{\phi}_{d}\right)+\sum_{e \in E^{\prime}} \int_{e}\left[r-r_{h}\right] \boldsymbol{\phi}_{d} \cdot \boldsymbol{n}_{e} d s \\
& \leq C h\left(h\|g\|_{0}+\left\|r-r_{h}\right\|_{1, h}\right)\left\|\boldsymbol{\phi}_{d}\right\|_{1} .
\end{aligned}
$$


Finally, we have the following estimate for the last term $I_{7}$ :

$$
\left|I_{7}\right| \leq C t^{2}\left\|p-p_{h}\right\|_{1}\left\|p_{d}-\pi_{1}^{h} p_{d}\right\|_{1} .
$$

Substituting inequalities (61)-(68) into (60), using the regularity (56) and the interpolation error estimate (57) and (51) with Theorem 6.5, we obtain

$$
\left(d, \phi-\phi_{h}\right) \leq C h^{2}\|d\|_{0}\|g\|_{0},
$$

which gives us

$$
\left\|\phi-\phi_{h}\right\|_{0} \leq C h^{2}\|g\|_{0} .
$$

Now, we turn to bound $\left\|\omega-\omega_{h}\right\|_{0}$. We first introduce the following problem: Find $\theta \in H_{0}^{1}(\Omega)$ such that

$$
(\nabla \theta, \nabla s)=(\phi, \nabla s), \quad \forall s \in H_{0}^{1}(\Omega) .
$$

Let $\bar{\theta}_{h} \in V_{h}^{n c}$ be the solution of the discrete problem

$$
\left(\nabla_{h} \bar{\theta}_{h}, \nabla_{h} s\right)=\left(\phi, \nabla_{h} s\right), \quad \forall s \in V_{h}^{n c} .
$$

It follows from Lemma 6.4 that

$$
\left\|\bar{\theta}_{h}-\theta\right\|_{0} \leq C h^{2}\|\phi\|_{1} \leq c h^{2}\|g\|_{0} .
$$

From (66), $\theta=\omega-\lambda^{-1} t^{2} r$. Denote $\theta_{h}=\omega_{h}-\lambda^{-1} t^{2} r_{h}$; by (18) and (70), we have

$$
\left(\nabla_{h}\left(\theta_{h}-\bar{\theta}_{h}\right), \nabla_{h} s\right)=\left(\phi_{h}-\phi, \nabla_{h} s\right), \quad \forall s \in V_{h}^{n c} .
$$

Setting $s=\theta_{h}-\bar{\theta}_{h}$,

$$
\left\|\nabla_{h}\left(\theta_{h}-\bar{\theta}_{h}\right)\right\|_{0} \leq C\left\|\phi_{h}-\phi\right\|_{0} \leq C h^{2}\|g\|_{0} .
$$

It follows from (59), (171) and (72) that

$$
\begin{aligned}
\|\omega-\omega\|_{0} & \leq\left\|\theta-\theta_{h}\right\|_{0}+\lambda^{-1} t^{2}\left\|r-r_{h}\right\|_{0} \\
& \leq\left\|\theta-\bar{\theta}_{h}\right\|_{0}+\left\|\theta_{h}-\bar{\theta}_{h}\right\|_{0}+\lambda^{-1} t^{2}\left\|r-r_{h}\right\|_{0} \\
& \leq C h^{2}\|g\|_{0} .
\end{aligned}
$$

Remark 6.8. We can extend our analysis to the element of [16] and obtain its optimal $L^{2}$ error estimate, which is missing in the literature.

Remark 6.9. To simplify the notation and fix the main idea, we present the analysis on the rectangular mesh. Obviously, both elements can be generalized to the general quadrilateral mesh. In addition, a similar argument herein shows that the energy error estimate is of order $h^{\alpha}$, and that the $L^{2}$ norm error estimate is of order $h^{2 \alpha}$, provided that the mesh satisfies the $(1+\alpha)$ section condition with $0 \leq \alpha \leq 1$ [17. This implies that the convergence rates in both norms depend on the mesh parameter $\alpha$. As a consequence, optimal error estimates hold only for mildly distorted meshes with $\alpha=1$. In [18, two nonconforming quadrilateral elements are proposed with optimal error estimates uniformly in $\alpha$ with respect to both energy norm and $L^{2}$ norm. 


\section{REFERENCES}

[1] D.N. Arnold, F. Brezzi, B. CockBurn and L.D. Marini, Unified analysis of discontinuous Galerkin methods for elliptic problems, SIAM. J. Numer. Anal., 2002 (39), pp. 1749-1779. MR.1885715 (2002k:65183)

[2] D.N. Arnold, F. Brezzi And L.D. Marini, A family of discontinuous Galerkin finite elements for Reissner-Mindlin plate, J. Sci. Comp., 2005 (22), pp. 25-41. MR2142189 (2006b:65160)

[3] D.N. ARnold And R.S. FAlK, A uniformly accurate finite element method for ReissnerMindlin plates, SIAM. J. Numer. Anal., 1989 (26), pp. 1276-1290. MR1025088 (91c:65068)

[4] K. J. Bathe, F. Brezzi And M. Fortin, Numerical approximation of Reissner-Mindlin plates, Math Comp., 1986 (47), pp. 151-158. MR842127(87g:73057)

[5] K. J. Bathe, F. Brezzi And M. Fortin, Mixed-interpolated elements for Reissner-Mindlin plates, Int. J. Num. Meths. Engreg., 1989 (28), pp. 1787-1801. MR1008138 (90g:73090)

[6] S.C. Brenner And L.R. Scott, The Mathematical Theory of Finite Element Methods. Springer-Verlag, 2nd Edition, 2002. MR1894376 (2003a:65103)

[7] S.C. Brenner, Poincare-Friedrichs inequalities for piecewise $H^{1}$ functions, SIAM. J. Numer. Anal., 2003 (41), pp. 306-324. MR.1974504 (2004d:65140)

[8] S.C. Brenner, Korn's inequalities for piecewise $H^{1}$ vector fields, Math.Comp., 2004 (73), pp. 1067-1087. MR2047078(2005c:65096)

[9] F. Brezzi And M. Fortin, Mixed and Hybrid Finite Element Methods, Springer-Verlag, 1991. MR.1115205 (92d:65187)

[10] F. BrezzI, M. Fortin AND R. Stenberg, Error analysis of mixed-interpolated elements for Reissner-Mindlin plate, Math. Models. Meth. Appl. Sci., 1991 (1), pp. 125-151. MR.1115287 (92e:73030)

[11] F. Brezzi And L.D. Marini, A nonconforming element for Reissner-Mindlin plate, Computers \& Structures, 2003 (81), pp. 515-522. MR2001877(2005f:74074)

[12] P.G. Ciarlet, The Finite Element Method for Elliptic Problems. North-Holland, 1978; reprinted as SIAM Classics in Applied Mathematics, 2002. MR 0520174 (58:25001)

[13] Hou DE Han, Nonconforming elements in the mixed finite element method, J. Comp. Math., 1984 (2), pp. 223-233. MR815417 (87d:65130)

[14] Jun Hu, Pingbing Ming And ZHONG-Ci SHi, Nonconforming quadrilateral rotated $Q_{1}$ element for Reissner-Mindlin plate, J. Comp. Math., 2003 (21), pp. 25-32. MR1974269|(2004c:65143)

[15] Q. Lin, L. ToBiska AND A. ZHou, On the superconvergence of nonconforming low order finite elements applied to the Poisson equation, IMA. J. Numer.Anal., 2005(25), pp. 160181. MR2110239 (2005k:65256)

[16] C. Lovadina, A lower-order nonconforming finite element for Reissner-Mindlin plates, SIAM. J. Numer. Anal., 2005 (42),pp. 2688-2705 . MR2139411(2006b:65172)

[17] Pingbing Ming and Zhong-Ci Shi, Quadrilateral mesh, Chinese Ann. Math. Ser. B., 2002 (23), pp. 235-252. MR1924140 (2003h:65163)

[18] Pingbing Ming And ZHOng-CI SHI, Two nonconforming quadrilateral finite elements for Reissner-Mindlin plate, Math. Model. Meth. Appl. Sci., 2005 (15), pp. 1503-1518. MR2168943 (2006g:74103)

[19] P. PEISKER and D. BRAEss, Uniform convergence of mixed interpolated elements for ReissnerMindlin plates, RAIRO. Anal. Numér., 1992 (26), pp. 557-574. MR1177387 (93j:73070)

[20] J. PitKÄRAnta, Analysis of some low-order finite element schemes for Mindlin-Reissner and Kirchhoff plates, Numer. Math., 1988 (53), pp. 237-254. MR.946378 (89f:65126)

[21] J. PitKÄrAnta AND M. SuRI Design principles and error analysis for reduced-shear plate bending finite elements, Numer. Math., 1996 (75), pp. 223-266. MR1421988 (98c:73078)

[22] R. Rannarcher and S. Turek, Simple nonconforming quadrilateral Stokes element, Numer. Meth. Part. Diff. Equations., 1992 (8), pp. 97-111. MR1148797 (92i:65170)

[23] L. R. ScOtT AND S. Zhang, Finite element interpolation of nonsmooth functions satisfying boundary conditions, Math. Comp., 1990 (54), pp. 483-493. MR.1011446 (90j:65021)

[24] Zhong-CI SHI, A convergence condition for quadrilateral Wilson element, Numer. Math., 1984 (44), pp. 349-361. MR757491 (86d:65151)

[25] Zhong-Ci SHI, The F-E-M-Test for nonconforming finite elements, Mathematics of Computation, 1987 (49), pp. 391-405. MR.906178 (88g:65120)

[26] R. Stenberg , Analysis of mixed finite element methods for the stokes problems: A unified approach, Math. Comp., 1984 (42), pp. 9-23. MR0725982 (84k:76014) 
[27] M. SuRI, I. BABuŠKa And C. SChWAB, Locking effects in the finite element approximation of plate models, Math. Comp., 1995 (64), pp. 461-482. MR1277772 (95f:65207)

[28] XIU Ye, A rectangular element for the Reissner-Mindlin plate, Numer. Meth. Part. Diff. Equations, 2000. MR.1740136 (2000j:74093)

No 55, Zhong-Guan-Cun Dong Lu, Institute of Computational Mathematics, Chinese Academy of Sciences, Beijing 100080, China

E-mail address: hujun@lsec.cc.ac.cn

Current address: LMAM and School of Mathematical Sciences, Peking University, Beijing 100871, China

E-mail address: hujun@math.pku.edu.cn

No 55, Zhong-Guan-Cun Dong Lu, Institute of Computational Mathematics, Chinese Academy of Sciences, Beijing 100080, China

E-mail address: shi@lsec.cc.ac.cn 